Der Hans-Janssen-Preis 2010 wurde Frau Kristin Böse, Köln, für ihre Arbeit „Gemalte Heiligkeit - Bildererzählungen neuer Heiliger in der italienischen Kunst des 14. und 15. Jahrhunderts"verliehen.

\title{
Die Kunst, Heilige zu machen. Zur Medialität und Funktion von Bildviten im Kult Francesca Romanas und anderer Religiosen im Tre- und im Quattrocento
}

\section{KRISTIN BÖSE}

Als im Jahre 1308 in der umbrischen Stadt Montefalco die Äbtissin des Konventes S. Croce verstorben war, veranlassten die Mitschwestern eine Obduktion. Zeitlebens hatte Chiara da Montefalco über körperliche Schmerzen geklagt, die sie auf eine Vision zurückführte, in deren Verlauf Christus ihr sein Kreuz in das Herz gepflanzt haben sollte. Bei der Obduktion kamen im Herzen der Verstorbenen ein Kruzifix und materialisierte Zeichen der Arma Christi, z. B. die Nägel, zum Vorschein. Diese wurden als Vergegenwärtigung von Christi Passion in Chiara und damit als sichtbare Beweise ihrer Heiligkeit gedeutet und trugen einige Jahre später zur Aufnahme eines Kanonisierungsverfahrens bei.

Der Obduktionsbericht rückt ein Thema in das Blickfeld, das die Kirche und deren Institutionen im

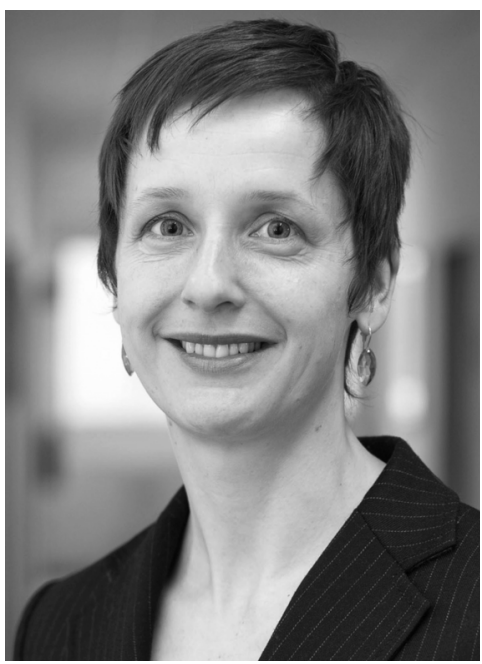

Kristin Böse,Wissenschaftliche Mitarbeiterin an dem Fritz-Thyssen-Forschungsprojekt „Ornament, Zeichen und Raum - Zur Konstruktion von Identität in nordspanischen Handschriften des 10.-12. Jh.“, Kunsthistorisches Institut der Universität zu Köln, Trägerin des Hans-Janssen-Preises 2010 ausgehenden Mittelalter vielfach beschäftigte: Wie lassen sich übersinnliche Erfahrungen verifizieren? Und wie lässt sich Unsichtbares in Wort und Bild fassen? Dabei ging es um nichts weniger, als über das zu urteilen, was dem Außenstehenden verschlossen blieb. Doch gerade dieser geheimnisvolle Widerspruch machte Visionärin- 
nen oder in Klausen lebende Religiosen wie Chiara da Montefalco, Francesca Romana, Giulia da Certaldo, Fina da San Gimignano, Caterina da Siena und andere vom späten 13. bis zum 15. Jahrhundert so verehrungswürdig. Die ursprünglich verschiedentlich als pinzoche oder monache di casa bezeichneten Frauen suchten außerhalb bestehender Ordensstrukturen nach religiösen Daseinsformen zwischen Kloster und Welt, Ehe und enthaltsamem Leben. Von ihnen haben sich schon bald nach dem Tod vielschichtige Bildensembles erhalten, die der Vermittlung von visionärem Erleben und von extremer asketischer Lebensweise dienten.

Obwohl die Religiosen zumeist nur lokal verehrt wurden, handelt es sich bei ihnen keinesfalls um ein randständiges Phänomen. Dies unterstreichen komplexe Bildtafel- und Wandmalereizyklen, ferner Reliquientabernakel und bemalte Holzsarkophage von insgesamt elf Religiosen, die in meiner Arbeit berücksichtigt wurden. Die bildliche Verehrung wurde ferner von weiten Teilen der städtischen Gesellschaft, von Pfarrgemeinden, Bruderschaften und jungen monastischen Gruppierungen getragen, die man, auf heutige soziale Strukturen übertragen, vielleicht als Mittelschicht bezeichnen würde. Was regte diese an, den Kult solch vermeintlich randständiger Existenzen zu fördern, und was für Erwartungen verbanden sich mit den Bildstiftungen?

Das räumliche Nebeneinander von Bildnarration und Grab bzw. Reliquien rückt insbesondere deren Zusammenspiel unter drei Gesichtspunkten in den Vordergrund: (1) Wie konnte auf der Bildebene etwa durch Raumanordnungen, (2) wie durch die Anordnung von Bildfeldern entlang von Blickachsen und schließlich (3) wie in Korrelation mit den Funktionen der Bestimmungsorte der Bilder ein entsprechender Kult etabliert werden.

Aufgrund heterogener Adressatenkreise und der daraus resultierenden, sich von Fall zu Fall verschiebenden inhaltlichen Gewichtungen wurden zwei Wege eingeschlagen: Am Beispiel der Bildzyklen zum Leben Francesca Romanas aus dem 15. Jahrhundert kann zunächst eine durch inhaltliche und bildmediale Überformungen gekennzeichnete Kultentwicklung über einen längeren Zeitraum verfolgt werden. Im Abgleich mit den verschriftlichten Fassungen der Vita und den Akten der erfolglosen Kanonisierungsverfahren spiegeln die Bildprogramme jene zum Teil widerstrebenden Intentionen wider, die zwischen der von Francesca gegründeten Oblatinnengemeinschaft, sodann der Olivetanerkongregation, der die Oblatinnen institutionell verbunden waren, und schließlich jenem an den Kanonisierungsverfahren beteiligten Kurienklerus bestanden.

Kurz nach Francescas Tod im Jahre 1440 entstand vermutlich für einen Seitenaltar ihrer Begräbniskirche S. Maria Nova auf dem Forum Romanum 
die erste Bildvita, von der sich einzelne Tafelbilder erhalten haben. Deren inhaltlicher Schwerpunkt liegt auf jenen Gottesbegegnungen, in welchen die Gründung der Gemeinschaft und ihre spirituellen Prämissen festgelegt und zugleich himmlisch legitimiert wurden. Hingegen waren die beiden nachfolgenden Wandmalereizyklen für den sich seit den 1430er Jahren durch systematischen Ankauf von Häusern formierenden Klosterkomplex „Tor de Specchi“ am Fuße des römischen Campidoglio bestimmt. Damit etablierte sich jenseits des Grabes ein weiterer Ort, mit dem sich durch Prozesse von Ein- und Überschreibungen mittels Inschriften und Bilder die Erinnerung an die Gründerin bis heute bewahrt hat.

Zwar gingen die schon bekannten Visionsdarstellungen aus dem ältesten Bildtafelzyklus in den 1468 fertiggestellten Freskenzyklus ein, wurden jedoch um Szenen ergänzt, die das thaumaturgische Wirken Francesca Romanas für die stadtrömische Öffentlichkeit belegen. Diese inhaltliche Verschiebung lässt sich einerseits als Strategie der Oblatinnen werten, ihre halboffene, d. h. nicht zur Klausur verpflichtete Lebensform nach außen zu vertreten, andererseits ist sie theologischen Diskussionen um die Verifizierung von Gottesbegegnungen geschuldet, die sich etwa auch an den Visionen Caterinas da Siena entzündeten. So wurden als Beleg übersinnlicher Erfahrungen etwa Wunderhandlungen gefordert. Innerhalb des Bildprogramms werden darüber hinaus durch die gezielte Anordnung von Bildfeldern verschiedene Perspektiven auf den mystischen Körper angeboten: Francescas Gottesbegegnungen werden durch Darstellungen flankiert, in denen Außenstehende den ekstatischen Körper der Religiosen bezeugen. Dennoch wurde die Oblatin im 15. Jahrhundert nicht mehr kanonisiert. Im Vergleich zum überregional erfolgreichen Kult der Dominikanerterziarin Caterina da Siena werden die bildmedialen Grenzen der mit einem Ort verbundenen Freskenzyklen deutlich. Ähnlich den Dominikanern versuchte die Olivetanerkongregation seit den 1440er Jahren durch die Produktion sparsam illuminierter Handschriften die Vita Francescas zu verbreiten. Doch schon bald schlief das Projekt ein. Schließlich gab es innerhalb der Kongregation niemanden, für den die verwitwete Francesca Romana hätte Vorbild sein können, da die Oblatinnen deren einzige assoziierte Frauengemeinschaft blieben.

Ausgehend von den Bildensembles im Kult Francesca Romanas, ergeben sich sodann Leitfragen, durch die das Bildmaterial anderer weiblicher Religiosen vergleichend gesichtet werden kann. Zunächst kennzeichnet das Nebeneinander verschiedener Perspektiven auf den visionären oder streng asketisch lebenden Körper, vermittelt durch Raum- und Blickbeziehungen, auch die Bildviten anderer weiblicher Religiosen. Als Beispiel dient 
eine Darstellung Giulias da Certaldo in ihrer Klause. Sie gehört zu einer Predella der zweiten Hälfte des 15. Jahrhunderts in der Kirche SS. Jacopo e Michele in Certaldo, an die einst die Klause angeschlossen war. Den Kindern, die zur Klause herbeieilen, um die Reklusin mit Nahrungsmitteln zu versorgen, und die im Gegenzug mit einem Blumenwunder belohnt werden, bleibt das Innere des Rückzugsortes verborgen. Selbst dem Betrachter, dem immerhin ein Blick auf den asketisch zugerichteten Körper erlaubt ist, erscheint das Innere der Klause völlig ungestaltet.

Diese Dialektik von Innen und Außen, Verbergen und Offenbaren korreliert auf der Ebene der Bildmedien mit aufklappbaren Reliquientabernakeln und bemalten Holzsarkophagen, durch die die Nähe zu den sterblichen Überresten kontrolliert werden konnte. So bringt etwa ein bemaltes Reliquientabernakel, das 1402 für das Grab Finas in der einstigen Pfarrkirche von San Gimignano entstanden ist und die Reliquienbüste der Religiosen enthielt, mit jenen den Bildgattungen eigenen Gestaltungsmitteln verschiedene Aspekte der Heiligkeit im Vorgang des Klappens der Türen zum Ausdruck. Wenn das Reliquiar gezeigt wurde, dann baute sich etwa ein Spannungsverhältnis zur Malerei auf dem rechten Seitenflügel auf, in der der von Mäusen gepeinigte und gelähmte Körper Finas zur Darstellung gelangt. Dagegen erscheint im Reliquiar das mädchenhafte Antlitz als Idealisierung zeitgenössischer Schönheit und Jungfräulichkeit und vermittelt durch die Verzierung mit Blattgold und Glasflüssen den Eindruck himmlischer Existenz und körperlicher Unversehrtheit.

Inwiefern und auf welche Weise mystisches Erleben und/oder Anachorese bildwürdig wurden, hing stets auch davon ab, ob die Religiosenkulte an einen Konvent oder an eine Pfarrkirche gebunden waren. Junge, im Institutionalisierungsprozess begriffene Religiosengemeinschaften leiteten aus den visionären Fähigkeiten der als heilig verehrten Klostergründerinnen ihre Existenzberechtigung ab und erwarteten spirituelle Anleitung. Dagegen knüpfte die städtische Pfarrgemeinde an das aus der Reklusion resultierende thaumaturgische Wirken an: Mit dem Rückzug der Reklusin wird zugleich deren spirituelle Präsenz erzeugt, die durch Wunderhandlungen an der Bürgerschaft offenbar wird. Wenn in der Bildvita Giulias da Certaldo die jeweiligen Leistungen der Reklusin als Fürbitterin und der sich kümmernden Kinder füreinander thematisiert werden, dann wird daran die jeweils für die andere Seite stellvertretend gelebte Teilhabe an der religiösen Sozialordnung erkennbar. Insgesamt fungierten die bildmedial ausdifferenzierten Ausstattungskonzepte als Vehikel für die Regulierung sozialer Beziehungen auf doppelte Weise: einmal auf der Ebene der Bildvita zwischen individueller und öffentlicher Frömmigkeit, d. h. zwischen der Religiosen 


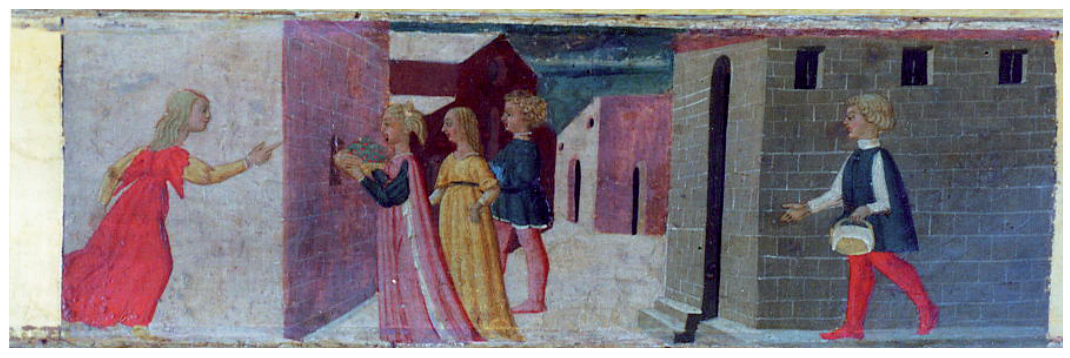

Abbildung 1: Giulia da Certaldo in ihrer Klause. Detail einer Predella, 2. Hälfte 15. Jahrhundert. Certaldo, SS. Jacopo e Michele

und einer städtischen Gemeinschaft; zum andern dienen die materiellen Bilder als Projektionsfläche, das Verhältnis zwischen Bildstiftern und sozialer Umgebung zu thematisieren.

Diese in den Bildern betonte enge Verflechtung zwischen einer bestimmten Gemeinschaft und ihrer Religiosen war nicht unbedingt geeignet, eine breitere Verehrung zu erzielen. Und es mag - nicht nur angesichts geringer finanzieller Mittel und Einflussmöglichkeiten - bezweifelt werden, dass eine Kanonisierung immer angestrebt wurde. Die Wirksamkeit der Bilder, die mitunter sogar schon vorlagen, bevor eine schriftliche Vitenfassung greifbar wird, ist dennoch nicht zu unterschätzen. Dass im Zuge erneuter Kanonisierungsversuche im 17. Jahrhundert gerade auch die ältesten Bildzeugnisse begutachtet wurden, belegt deren Vermögen, die informelle Verehrung über einen langen Zeitraum aufrechtzuerhalten.

\section{Auswablbibliographie}

Barone, Giulia/Zarri, Gabriella u. a. (Hgg.), Donne e fede. Santità e vita religiosa in Italia (Storia delle donne in Italia), Rom 1994.

Bornstein, Daniel/Rusconi, Roberto (Hgg.), Women and Religion in Medieval and Renaissance Italy, 2. Aufl. Chicago/London 1996.

Ganz, David, Medien der Offenbarung. Visionsdarstellungen im Mittelalter, Berlin 2008.

Gill, Katherine J., Penitents, pinzochere, and mantellate: Varieties of Women's Religious Communities in Central Italy, c. 1300-1520 (UMI No. 94-10101), Diss. Ann Arbor 1994.

Hamburger, Jeffrey, Seeing and Believing. The Suspicion of Sight and the Authentification of Vision in Late Medieval Art and Devotion, in: Krüger, Klaus/Nova, Alessandro (Hgg.), Imagination und Wirklichkeit. Zum Verhältnis von mentalen und realen Bildern in der Kunst der frühen Neuzeit, Mainz 2000, S. 47-69. 
Krüger, Klaus, Bildandacht und Bergeinsamkeit. Der Eremit als Rollenspiel in der städtischen Gesellschaft, in: Blume, Dieter/Belting, Hans (Hgg.), Malerei und Stadtkultur in der Dantezeit. Die Argumentation der Bilder, München 1989, S. 187-200.

Thomas, Anabel, Art and Piety in the Female Religious Communities of Renaissance Italy. Iconography, Space, and the Religious Women's Perspective, Cambridge 2003.

Wenderholm, Iris, Bild und Berührung. Skulptur und Malerei auf dem Altar der italienischen Frührenaissance (Italienische Forschungen des Kunsthistorischen Instituts in Florenz Max-Planck-Institut: I Mandorli 5), Berlin 2006. 